\title{
Sistema de classificação de pacientes em assistência domiciliária*
}

\author{
Patients classification system in home care
}

Sistema de classificación de pacientes en atención domiciliaria

\section{Luiza Watanabe Dal Ben ${ }^{1}$, Raquel Rapone Gaidiznski ${ }^{2}$}

\section{RESUMO}

Objetivos: O objetivo deste artigo é apresentar os Sistemas de Classificação de Pacientes (SCP) existentes nessa área. Métodos: revisão bibliográfica. Resultados: Os instrumentos encontrados foram: de Garrard et al, Home Health Care Classification System4.4 Método Home Health Care Classification System (HHCC), segundo Saba (1991), Escala Katr, as tabelas utilizadas pela Associação Brasileira de Empresas de Medicina de Internação Domiciliar e pelo Núcleo Nacional das Empresas de Assistência Domiciliar, o TISS-Intermediário adaptado por Dal Ben e o catálogo de prestação de serviços da "Fondation des Services d'aide et de Soins à Domicile". Desses instrumentos os dois últimos mensuram a carga de trabalho e os demais apresentam indicadores para obter recursos humanos e financeiros. Considerações finais: Quanto ao tempo despendido na assistência ao paciente considera-se como dependência total - 24 horas; dependência parcial - 12 horas; dependência moderada -6 horas de assistência / dia / paciente.

Descritores: Serviços de enfermagem/rganização \& administração; Pessoal de saúde; Cuidados domiciliares de saúde; Recursos Humanos de Enfermagem.

\begin{abstract}
Objective: This article aims at presenting the existing patient classifications systems. Methods: a literature review. Results: Instruments found out are: that by Garrard et al, Home Health Care Classification System, Katz Scale, the utilized tables by the Brazilian Medical Enterprises in Home Care and by Home Care National Enterprises Center, the Therapeutic Intervention Scoring System (TISS) for non-ICU patients, adapted by Dal Ben, and the catalog for services delivery of the Assistance and Home Care Services Foundation (Fondation des services d'aide et des soins à domicile). From these instruments, the last two mentioned measure the workload and others present indicators for obtaining human and financial resources. Final considerations: In relation to the time spent in caring patients, it is of 24 hours when dependence is total; of 12 hours when it is partial and moderated dependence -6 hours caring/per day, per patient.
\end{abstract}

Keywords: Nursing services/administration \& organization; Health Personnel; Home Nursing; Nursing Staff.

\section{RESUMEN}

Objetivo: el objetivo de este articulo es presentar los sistemas de clasificación de pacientes existentes en esta area. Metodos: revisión bibliográfica. Resultados: Los instrumentos encuentrados fueron: de Garrard et al, Home Health Care Classification System, Escala Katz, las tablas utilizadas por la Asociación Brasileña de Empresas de Medicina de Atencion Domiciliaria y por el Nucleo Nacional de Empresas de Atención Domiciliaria, el TISS-Intermediário adaptado por Dal Ben y el catálogo de la prestación de servicios de la "Fundación de servicios de ayuda y atención domiciliária”. De eses instrumentos los dos ultimos miden la carga del trabajo y los demás presentan indicadores para obtener los recursos humanos y financieros. Consideraciones Finales: Cuanto al tiempo gasto en la atención del paciente se considera de 24 horas para los que tienen dependencia total, de 12 horas para los que tienen dependencia parcial y de 6 horas por dia/por paciente para los que tienen dependencia moderada.

Descriptores: Servicios de Enfermería/organización \& administración; Personal de Salud; Cuidados Domiciliarios de Salud; Personal de Enfermería.

\footnotetext{
* Este artigo é parte da tese de Doutorado intitulada "Dimensionamento do pessoal de enfermagem em assistência domiciliária: percepção de gerentes e enfermeiras ", apresentada no Programa de Pós-graduação em Enfermagem da Escola de Enfermagem da Universidade de São Paulo - USP - São Paulo (SP), Brasil.

${ }^{1}$ Mestre em Enfermagem pela Escola de Enfermagem da Universidade de Paulo - USP - São Paulo (SP), Brasil.

2 Professor Livre-Docente da Escola de Enfermagem da Universidade de São Paulo - USP - São Paulo (SP), Brasil.
} 


\section{INTRODUÇÃO}

O desempenho da prática de enfermagem na assistência domiciliária (AD), uma nova e crescente área de atuação da enfermeira no Brasil, exige conhecimento relacionado à responsabilidade ética, profissional e suas implicações legais no exercício da enfermagem ${ }^{(1)}$.

Os parâmetros do quadro de profissionais de enfermagem nas instituições de saúde e assemelhados, estabelecendo o quantiqualitativo mínimo dos diferentes níveis de formação dos profissionais de enfermagem à cobertura assistencial nas instituições de saúde estão na Resolução COFEN no 293/2004(2). Embora, se possa entender os assemelhados, essa Resolução não contempla o dimensionamento do pessoal de enfermagem aos ambulatórios e em AD.

Para a realização do planejamento e a avaliação do quantitativo de recursos humanos de enfermagem é preciso ter o conhecimento das variáveis envolvidas no processo de dimensionar o pessoal de enfermagem. Uma das etapas é a identificação da carga média de trabalho diária ${ }^{(3-4)}$. A carga de trabalho consiste no produto da quantidade média diária de pacientes/clientes assistidos, conforme o grau de dependência da equipe de enfermagem ou do tipo de intervenção pelo tempo médio de assistência de enfermagem utilizado, por cliente, de acordo com o grau de dependência ou intervenções realizadas ${ }^{(3-4)}$.

O desempenho da equipe de enfermagem está diretamente relacionado com a adequação do tempo de permanência do profissional de enfermagem no domicílio, de acordo com as necessidades do paciente, seu perfil pessoal e profissional; à viabilidade dos custos $\mathrm{da}$ assistência e à supervisão mantida pela enfermeira ${ }^{(5-6)}$.

Para tanto, o Sistema de Classificação de Pacientes (SCP), entendido como uma forma de determinar o grau de dependência de um paciente em relação à equipe de enfermagem, objetiva estabelecer o tempo despendido no cuidado direto e indireto, bem como o qualitativo de pessoal, para atender às necessidades biopsicossocioespirituais do paciente ${ }^{(3)}$.

A partir da década de 60 do século XX, a literatura internacional e nacional tem apresentado inúmeros SCP como fundamentação da carga de trabalho em enfermagem, sobretudo para pacientes internados em hospitais.

Considerando a importância do tema, a presente pesquisa tem o objetivo de realizar uma revisão bibliográfica para identificar os SCP existentes na área de assistência domiciliária.

\section{MÉTODOS}

Para a coleta de dados foi realizado um levantamento bibliográfico, no período de 1970 a 2004, nas bases “ SciELO", “ MEDLINE”, " LILACS", "Web of
Science", em catálogos de serviços e junto às associações de AD no Brasil. As associações e sociedades consultadas foram: Associação Brasileira de Home Health Care (ABRAHHCARE $^{(7)}$ ), criada em julho de 1995; Associação Brasileira de Empresas de Medicina de Internação Domiciliar (ABEMID ${ }^{(8)}$ ), fundada em 1997; Sociedade Brasileira de Enfermagem em Home Care $\left(\mathrm{SOBEHC}^{(9)}\right)$, fundada em fevereiro de 2000; Núcleo Nacional das Empresas de Assistência Domiciliar $\left(\mathrm{NEAD}^{(10)}\right)$, criado em 2003.

\section{RESULTADOS}

No levantamento bibliográfico, o estudo de Inui et al. ${ }^{(11)}$, identifica a adequação das indicações da equipe de assistência domiciliária de um hospital para pacientes ao programa de AD. Conclui que a técnica de avaliação apresentada, embora confiável, demandava um custo elevado, pela exigência de juízes, enfermeiras com habilidades clínicas e com experiência relevante anterior.

$\mathrm{O}$ instrumento desenvolvido por Garrard et al ${ }^{(12)}$, em 1987, em forma de "check list" permite reconhecer o perfil do paciente que necessita assistência de enfermagem no domicílio, no momento da alta hospitalar, mas não quantifica a demanda do cuidado no momento da alta hospitalar. Esse instrumento é dividido em três categorias: problemas de saúde/médico; problemas psicossociais/comportamentais e educação/coordenação da necessidade de cuidados. Desses vinte e cinco itens, se um item for assinalado, demonstra a indicação do paciente ao serviço de AD.

O Home Health Care Classification System $(\mathrm{HHCC})^{(13)}$ Classificação do Cuidado em Saúde Domiciliar de Diagnósticos e Intervenções de Enfermagem, é um método para acessar e classificar pacientes, a fim de determinar os recursos necessários para fornecer serviço de atendimento domiciliário à população com seguro saúde Medicare (USA) e que, inclui os resultados do cuidado prestado. Esta classificação foi conduzida por Saba ${ }^{(13)}$ na Escola de Enfermagem da Georgetown University e, foi traduzida ao português por Heimar F Marin. O estudo contribui para a identificação das atividades desenvolvidas em assistência domiciliária, auxiliando no cotidiano da prática da AD. Cada componente de enfermagem é definido como um conjunto de elementos que representa o padrão funcional, comportamental ou fisiológico do cuidado de saúde em domicílio, relatando um padrão único da prática clínica de enfermagem em saúde comunitária ${ }^{(13)}$.

No HHCC, as intervenções de enfermagem são definidas como ação de enfermagem, tratamento, procedimento ou atividade designada a atingir um resultado para o diagnóstico médico ou de enfermagem, pelo qual a enfermeira é responsável. As descrições, quanto ao tipo de ação de enfermagem para cada 
intervenção são respectivamente: a) avaliar (assessment) o dado coletado e analisar o estado de saúde; b) cuidado direto é o desempenho de ação terapêutica; c) ensinar é prover conhecimento e habilidade; d) gerenciamento é coordenar e encaminhar ${ }^{(13)}$.

Na Suíça, o grupo de estudo formado, em 1992, pelos profissionais dos cantões da Suíça Romande, Berne e Tessin, do Institut Suisse de la Santé Publique (ISP) ${ }^{(14)}$, definiu e publicou seu processo de investigação para mensurar a carga de trabalho nos cuidados na assistência em domicílio nos territórios citados. Nessa pesquisa, são identificadas as contribuições dos instrumentos Escala Katz e Home Health Care Classification System (HHCC), no processo de gestão, administração e qualidade da assistência prestada. A influência da utilização dos mesmos e o impacto desses nos níveis decisórios, envolvendo os profissionais, a política e, conseqüentemente, as fases de planejamento, organização, produtividade e de controle. $\mathrm{O}$ método Escala Katz avalia a evolução da autonomia e independência funcional do paciente; foi desenvolvido pelo Professor Sidney Katz e sua equipe, na Universidade de Columbia (EUA), é constituído do "Index of independence in Activity of Daily Living" (ADL), com o objetivo de avaliar o desempenho individual em relação às atividades básicas de vida diária (AVD) ${ }^{(14)}$.

$\mathrm{O}$ instrumento Intermediate TISS - therapeutic intervention scoring system for non-ICU patients, apresentado por Cullen, Nemeskal e Zaslavsky ${ }^{(15)}$, foi adaptado para identificar as intervenções presentes nos pacientes atendidos em AD. O instrumento foi traduzido para a Língua Portuguesa, como TISS - Intermediário e para obter o consenso de especialistas, considerados juízes, foi utilizada a Técnica Delphi em três etapas. Os juízes foram enfermeiras com experiência de 2 até 13 anos em $\mathrm{AD}$, na cidade de São Paulo, que atuavam na determinação do quantitativo de horas diárias de assistência de enfermagem domiciliária no momento da alta hospitalar. Esse estudo considerou a estreita relação entre estado de gravidade do paciente e a relação com o tempo de enfermagem necessário para o seu atendimento ${ }^{(5-6)}$.

O resultado obtido foi um instrumento composto de 104 intervenções, sendo 50 mantidas do TISS Intermediário e 54 novas intervenções que os juízes apontaram, como importantes na determinação do tempo de assistência de enfermagem domiciliária. As cinqüenta e quatro novas intervenções foram classificadas, de acordo com os domínios propostos por Nursing Interventions Classification (NIC), sendo 30 de domínio fisiológico básico, 13 de domínio fisiológico complexo, três de domínio comportamental, duas de domínio segurança, duas de domínio família, quatro de domínio sistema de saúde. Todas as novas intervenções apontadas são específicas da enfermagem e peculiares à assistência de enfermagem domiciliária. Desse instrumento, 46 itens são classificados como atividades conjugadas porque permitem a execução de outras intervenções ao mesmo tempo; 58 itens são denominados atividades isoladas por não possibilitarem a execução de outras atividades concomitantes. O instrumento adaptado foi publicado em 2004, na Revista da Escola de Enfermagem da USP(5-6).

A ABEMID recomenda a todas as empresas associadas o emprego dos instrumentos: Tabela de avaliação de complexidade assistencial - ABEMID (Anexo 1) e da Tabela de avaliação socioambiental ABEMID (Anexo 2) para a classificação dos pacientes. Esses instrumentos têm sido empregados sobretudo por uma das empresas líderes pela fundação da ABEMID. Essas tabelas são aceitas pelos planos de saúde do setor privado que ao classificarem os pacientes em alta complexidade, não geram muitos questionamentos por parte dos auditores dos planos de saúde. As autoras possuem a autorização do presidente da ABEMID, Dr. Josier Vilar para divulgar na tese de doutorado, as tabelas utilizadas pela associação.

A classificação dos pacientes, em alta complexidade, apontada na tabela ABEMID, como acima de 19 pontos e 24 horas de assistência de enfermagem, pelo alto grau de dependência de cuidados, refere-se a pacientes que necessitam dos mesmos aparatos utilizados durante sua internação hospitalar em unidades de cuidados intensivos e semi-intensivos. Assim, ao serem transferidos ao domicílio precisam durante sua internação domiciliária do mesmo quantitativo de 24 horas de assistência de enfermagem e de uma equipe multiprofissional. Os aspectos relacionados ao ambiente sociofamiliar apresentado no Anexo 2 estão sendo submetidos à apreciação da ABEMID para sua validação clínica pelas empresas associadas, em função da importância do contexto sociofamiliar na indicação da AD.

O NEAD adota outro SCP, sendo utilizado por uma das empresas que liderou a sua fundação. Os instrumentos apresentam pontuações distribuídas por grupos de indicadores, que de acordo com o quantitativo de pontos, classificam o paciente para avaliar a sua indicação para internação domiciliária de acordo com o quantitativo de horas de assistência de enfermagem (visitas, 6, 12 e 24 horas) (Anexo 3), ou a manutenção do paciente em internação domiciliária (Anexo 4). O NEAD também está solicitando às empresas associadas a validação clinica dos instrumentos. As autoras possuem a autorização do presidente do NEAD, Dr. Ari Bolonhesi para divulgar na tese de doutorado as tabelas utilizadas pelo núcleo.

Os instrumentos de classificação de pacientes utilizados pelas associações apresentados nos Anexos 1-4 verificam a presença de intervenções nos pacientes que demandam cuidados de enfermagem, demonstrando que existe um movimento para se provar a necessidade de classificar o paciente para o dimensionamento de pessoal de 
enfermagem domiciliária.

Em Genebra, a "Fondation Des Services D'aide Et De Soins À Domicile”- (FSASD) ${ }^{(16)}$, a partir de 2004, utiliza um catálogo de prestação e tratamento que direciona a prática profissional de cuidados de ajuda em domicílio. Para cada procedimento, há um código, uma descrição do que consiste o cuidado de ajuda a ser ministrado ao paciente, a freqüência em que é oferecido se semanal ou mensal, e a mensuração do tempo previsto para execução dos cuidados é apresentada em minutos. Por exemplo, a realização de banho completo do paciente no leito recebe o código 101, e os passos são: o preparo do banho e a retirada da roupa, banhar o cliente, aplicar uma loção protetora de pele, vestir o cliente, pentear os cabelos e refazer o leito. O procedimento tem a cobertura do seguro de saúde do governo (LAMAL), uma vez por dia em todos os dias da semana, o tempo despendido é de 40 minutos. Esse catálogo está apresentado como Anexo na tese de doutorado da autora.

Os cuidados em domićlilio são classificados, como cuidados de enfermagem, cuidados básicos e cuidados de ajuda em domicílio, sendo tais cuidados classificados em ações sociais, ações familiares e outras prestações de cuidados $^{(14)}$. Os cuidados básicos são realizados por profissionais com conhecimentos obtidos de formação básica, semelhantes ao técnico de enfermagem do Brasil. Os cuidados de ajuda em domićlíio são oferecidos, em especial, aos idosos e incluem a estrutura de manutenção das atividades de vida diária todos os dias da semana e, atualmente, está sendo estendido a 24 horas por dia.

Cabe lembrar que é competência dos enfermeiros em Genebra, orientarem somente os familiares e os pacientes em técnicas exclusivas de enfermagem, sendo proibida essa prática a pessoas contratadas pelas famílias que utilizarão desse conhecimento para fins empregatícios.

\section{CONSIDERAÇÕES FINAIS}

Ao se comparar o tempo despendido aos cuidados de enfermagem no catálogo utilizado em Genebra, pela FSASD, com o TISS-Intermediário adaptado por Dal Ben ${ }^{(5)}$ e Dal Ben, Sousa ${ }^{(6)}$, observa-se que o tempo é semelhante.

O tempo médio despendido no cuidado, conforme o grau de dependência do paciente e segundo os instrumentos de classificação pode-se considerar como: dependência total - 24 horas de assistência / dia / paciente; dependência parcial - 12 horas de assistência / dia / paciente; dependência moderada -6 horas de assistência / dia / paciente.

O TISS-Intermediário adaptado para a realidade da cidade de São Paulo e o catálogo utilizado em Genebra pela FSASD são instrumentos que apresentam a mensuração do tempo despendido na realização de cuidados de enfermagem em AD. Assim, podem ser utilizados como fonte na identificação da carga de trabalho e subsidiar o dimensionamento de pessoal de enfermagem em AD.

\section{REFERÊNCIAS}

1. Perspectivas sobre os rumos da enfermagem Oguisso T, Schmidt MJ. O exercício da enfermagem: uma abordagem ético-legal. São Paulo: LTr; 1999. p. 17185.

2. Conselho Federal de Enfermagem. Resolução 293, de 21 de setembro de 2004. Fixa e Estabelece Parâmetros para o Dimensionamento do Quadro de Profissionais de Enfermagem nas Unidades Assistenciais das Instituições de Saúde e Assemelhados [texto na internet]. Rio de Janeiro; 2004. [citado 2005 Jun 5] Disponível em: http://www.corensp.org.br/ resoluções/Resolução293.htm

3. Gaidzinski RR. Dimensionamento do pessoal de enfermagem segundo a percepção de enfermeiras que vivenciam essa prática [tese ]. São Paulo: Escola de Enfermagem da Universidade de São Paulo; 1994.

4. Gaidzinski RR, Fugulin FMT, Castilho V. Dimensionamento de pessoal de enfermagem em instituições de saúde. In: Kurcgant P. Gerenciamento de enfermagem. Rio de Janeiro: Guanabara Koogan; 2005. p.125-37.

5. Dal Ben LW. Instrumento para dimensionar horas diárias de assistência de enfermagem residencial [tese]. São Paulo: Escola de Enfermagem da Universidade de São Paulo; 2000.

6. Dal Ben LW, Sousa RMC. Adaptação de instrumento para dimensionar horas diárias de assistência de enfermagem residencial. Rev Esc Enferm. USP. 2004; 38(1):80-9.

7. Polianna P. A especialização faz o profissional [entrevista]. Rev Negócios e Soluções em Home Care. 2002; 3(15):3-6.

8. Vilar JM. Visão da ABEMID sobre o cenário atual de assistência à saúde privada no Brasil. Rev Bras Home Care. 2003; 8(97):4. .

9. SOBEHC - Sociedade Brasileira de Enfermagem Home Care - www.abesenacional. com.br/ outras.html - acesso em 15/05/2005achei o site: http://www.abesenacional.com.br/so outras.html

10. NEAD - Núcleo Nacional de Empresas de Assistência Domiciliar-nead.saude@ itelefonica.com.br

11. Inui TS, Stevenson KM, Plorde D, Murphy I. Needs assessment for hospital-based home care services. Res Nurs Health.1980; 3(3):101-6.

12. Garrard J, Dowd BE, Dorsey B, Shapiro J. A checklist to assess the need for home health care: instrument development and validation. Public Health Nurs. 1987; 4 (4):212-8.

13. Classificação do cuidado em saúde domiciliar 
(HHCC) de Diagnósticos e Intervenções de Enfermagem [texto $\mathrm{n}$ the Internet]. Traduzido por Marin HF. São Paulo: Centro de Informática em Enfermagem; 1991 [cited 2005 Jan 5] Disponível em: $<$ http://www.sabacare.com/portuguesetrans.html $>$ [Texto original: Saba V. Home Health Care Classification (HHCC), Of Nursing Diagnoses And Intervetions].

14. Institut Suisse Santé Publique. Aide et soins à domicile en Suisse romande, Berne et Tessin: Mesure de la charge de travail. Lausanne,IFG/ISP;1996.

15. Cullen DJ, Nemeskal AR, Zaslavsky AM. Intermediate TISS: a new Therapeutic Intervention Scoring System for non-ICU patients. Crit Care Med. 1994; 22(9):1406-11.

16. Foundation dês Services d'Aide et de Soins à Domicile FSASD - Aide et soins à domicile - De vou à nous Genève [manual].

Anexo I - Tabela de complexidade assistencial - Fonte: ABEMID, 2004.

TABELA DE AVALIAÇÃO DE COMPLEXIDADE ASSISTENCIAL - ABEMID ${ }^{4}$

Efetuada em:

/ 1

\begin{tabular}{|c|c|c|}
\hline \multicolumn{2}{|l|}{ Nome do Paciente: } & Complexidade: \\
\hline Idade: & \multicolumn{2}{|c|}{ Programação em dias de atendimento $-24 \mathrm{~h}:$} \\
\hline Convênio: & \multicolumn{2}{|c|}{ Programação em dias de atendimento - $12 \mathrm{~h}:$} \\
\hline Matrícula: & \multicolumn{2}{|c|}{ Programação em dias de atendimento - 06 h: } \\
\hline \multicolumn{3}{|l|}{ Diagnóstico Principal: } \\
\hline \multicolumn{3}{|l|}{ Diagnóstico Secundário: } \\
\hline Descrição & Itens da Avaliação & Pontos Atribuídos \\
\hline \multirow[t]{9}{*}{ Suporte Terapêutico } & Sonda Vesical Permanente & 1 \\
\hline & Sonda Vesical Intermitente & 2 \\
\hline & Traqueostomia sem Aspiração & 2 \\
\hline & Traqueostomia com Aspiração & 5 \\
\hline & Aspiração de Vias Aéreas Sup. & 3 \\
\hline & Acesso Venoso Prof. Continuo & 5 \\
\hline & Acesso Venoso Intermitente & 4 \\
\hline & A. Venoso Periférico Continuo & 5 \\
\hline & Diálise Domiciliar & 5 \\
\hline \multirow[t]{4}{*}{ Quimioterapia } & Oral & 1 \\
\hline & Subcutânea & 3 \\
\hline & Intravenosa & 5 \\
\hline & Intratecal & 5 \\
\hline \multirow[t]{4}{*}{ Suporte ventilatório } & $\mathrm{O}^{2}$ Intermitente & 2 \\
\hline & $\mathrm{O}^{2}$ Contínuo & 3 \\
\hline & Ventilação Mec. Intermitente & 4 \\
\hline & Ventilação Mecânica Contínua & 5 \\
\hline \multirow[t]{4}{*}{ Lesão Vascular/Cutânea } & Úlcera de Pressão Grau I & 2 \\
\hline & Úlcera de Pressão Grau II & 3 \\
\hline & Úlcera de Pressão Grau III & 4 \\
\hline & Úlcera de Pressão Grau IV & 5 \\
\hline \multirow{3}{*}{$\begin{array}{l}\text { Grau de Atividade da Vida Diária } \\
\text { Relacionada a Cuidados Técnicos }\end{array}$} & Independente & 0 \\
\hline & Semi-Dependente & 2 \\
\hline & Dependente Total & 5 \\
\hline \multirow{2}{*}{$\begin{array}{l}\text { Dependência de Reabilitação } \\
\text { Fisioterapia / Fonoaudiologia / Etc. }\end{array}$} & Independente & 0 \\
\hline & Dependente & 2 \\
\hline \multirow[t]{5}{*}{ Terapia Nutricional } & Suplementação Oral & 1 \\
\hline & Gastrostomia & 2 \\
\hline & SNE & 3 \\
\hline & Jejuno Íleo & 3 \\
\hline & Nutrição Parenteral Total & 5 \\
\hline
\end{tabular}

${ }^{4}$ Divulgação autorizada pelo presidente da ABEMID, Dr. Josier Vilar 


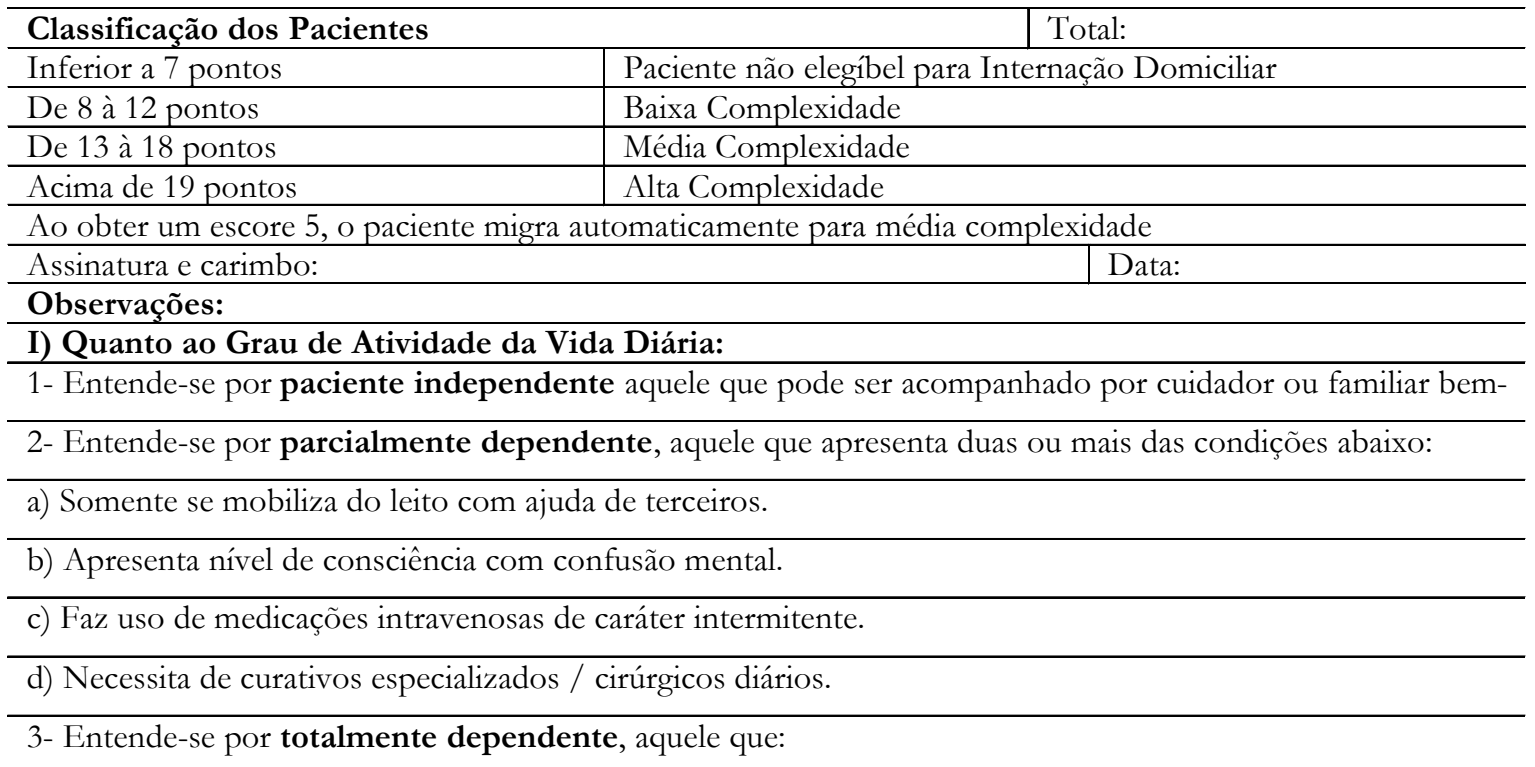

a) apresentam-se em prótese ventilatória contínua ou intermitente com três ou mais intervenções diárias.

b) apresenta-se incosciente / comatoso ou totalmente restrito ao leito, associado à necessidade de algum dos suportes terapêuticos: cateter vesical, traqueostomia, acesso venoso e diálise domiciliar.

c) faz uso de medicações intravenosas de caráter contínuo.

d) possui cirurgia de fixação da coluna, em decorrência de instabilidade grave, com menos de 60 dias de P.O.

II) Quanto à Classificação:

a) Se o somatório de pontos obtidos for menor ou igua a sete pontos, o paciente será considerado não elegíbel para iniciar ou manter-se no programa de internação domiciliar.

b) Se o somatório de pontos obtidos for de 8 a 12 pontos, o paciente será considerado de Baixa

c) Se o somatório de pontos obtidos for de 13 a 18 pontos, o paciente será considerado de Médica

d) Se o somatório de pontos obtidos for igual ou superior a 19 pontos, o paciente será considerado de Alta

e) Ao obter uma pontuação cinco, o paciente migra automaticamente para Média Complexidade.

f) Ao obter dois ou mais pontuações cinco, o paciente migra automaticamente para Alta Complexidade, independente do total de pontos obtidos (com cuidados de enfermagem de 24 horas). Obs.1 - Em TODOS os itens de avaliação, EXCETO os relacionados à coluna SUPORTE

TERAPÊUTICO, os pontos NÃO se somam, SEMPRE prevalecendo o item de MAIOR pontuação em Obs.2 - Entende-se por DEPENDÊNCIA TOTAL DE CUIDADOS a necessidade de enfermagem $24 \mathrm{~h}$.

Obs.3 - Entende-se por DEPENDÊNCIA PARCIAL DE CUIDADOS a necessidade de enfermagem $12 \mathrm{~h}$. 
Anexo II - Tabela de avaliação socioambiental - Fonte: ABEMID, 2004.

TABELA DE AVALIAÇÃO SÓCIO-AMBIENTAL - ABEMID ${ }^{5}$

1) AVALIAÇÃO SOCIAL

\begin{tabular}{l|l|c}
\hline A) Estrutura Familiar: \\
\hline a) & Núcleo familiar não identificado e sem condições de estruturação do cuidado & 0 \\
\hline b) & Núcleo familiar não identificado mas com condições de estruturação do & 2 \\
\hline c) & Núcleo familiar identificado, apóia a ID, mas não deseja assumir o cuidado & 5 \\
\hline d) & Núcleo familiar identificado, apóia a ID, e responsabiliza-se pelo cuidado & 10 \\
\hline
\end{tabular}

B) Consentimento e Participação Familiar

\begin{tabular}{l|l|c}
\hline a) & Família não aceita a desospitalização & 0 \\
\hline b) & Família aceita a ID, mas resiste por dificuldades financeiras e estruturais & 2 \\
\hline c) & Família aceita a ID, mas resiste por insegurança com o processo & 5 \\
\hline d) & Família apóia integralmente a desospitalização & 10 \\
\hline
\end{tabular}

C) Identificação e Treinamento do Cuidador

\begin{tabular}{l|l|c}
\hline a) & Não aceita "Cuidador" (familiar ou profissional) & 0 \\
\hline b) & Aceita "Cuidador" mas não tem recursos para assumir & 2 \\
\hline c) & Aceita "Cuidador" e familiar assumirá o cuidado & 2 \\
\hline d) & Aceita "Cuidador" e financiará o trabalho o "Cuidador” & 10 \\
\hline
\end{tabular}

\section{INTERPRETAÇÃO DO ESCORE}

\begin{tabular}{l|l}
\hline 1) Somatório dos itens $A+B+C$ atinge 0 e 6 pontos: & Pacientes NÂO elegível para ID \\
\hline 2) Somatório dos itens $A+B+C$ atinge 7 a 15 pontos: & Paciente é elegível com rigoroso \\
\hline 3) Somatório dos itens A+B+C atinge 16 ou mais pontos: & Paciente totalmente elegível para ID \\
\hline 2) AVALIAÇÃO AMBIENTAL & \multicolumn{2}{c}{ Pontuação }
\end{tabular}

\begin{tabular}{l|l|c}
\hline A) Espaço Físico & 0 \\
\hline a) & Residência não possui cômodo específico para receber o paciente & 2 \\
\hline b) & Residência possui cômodo inadequado e com limitadas condições de reestruturação & 5 \\
\hline c) & Residência possui cômodo específico, mas necessita de reformas simples para sua & 5 \\
\hline d) & Residência possui cômodo totalmente adequado para receber o paciente & 10 \\
\hline
\end{tabular}

\section{B) Acesso Físico}

\begin{tabular}{l|l|c}
\hline a) & Residência sem elevador e a escada não permite subir a maca & 0 \\
\hline b) & Residência sem elevador mas a escada permite subir a maca & 5 \\
\hline c) & Residência com elevador e sem dificuldade de acesso & 10 \\
\hline
\end{tabular}

C) Segurança e Meio Ambiente

\begin{tabular}{l|l|l}
\hline a) & Freqüente falta de água & Não recomendável ID \\
\hline b) & Freqüente falta de luz & Não recomendável ID \\
\hline c) & Esgoto não ligado à rede pública & Não recomendável ID \\
\hline d) & Local considerado de alto risco de segurança & Não recomendável ID \\
\hline e) & Distante mais de $100 \mathrm{Km} \mathrm{da} \mathrm{Central} \mathrm{de} \mathrm{Atendimento}$ & Não recomendável ID \\
\hline f) & Ausência de telefone no local da ID & Não recomendável ID \\
\hline
\end{tabular}

INTERPRETAÇÃO DO ESCORE

\begin{tabular}{l|c}
\hline 1) Somatório dos itens A+B entre 0 e 2 pontos & Paciente NÃO elegível para ID \\
\hline 2) Somatório dos itens A+B entre 3 e 7 pontos & Paciente elegível condicionalmente \\
\hline 3) Somatório dos itens A+B acima de 8 pontos & Paciente totalmente elegível para \\
\hline OBS: A identificação de qualquer item de segurança e meio ambiente, leva a condição de elegibilidade para \\
\hline
\end{tabular}

${ }^{5}$ Divulgação autorizada pelo presidente da ABEMID, Dr. Josier Vilar. 
Anexo III - Tabela de avaliação para internação domiciliar - Fonte: NEAD, 2004.

\section{TABELA DE AVALIAÇÃO PARA INTERNAÇÃO DOMICILIAR - NEAD ${ }^{6}$}

Paciente:

Convênio:

\section{GRUPO 1}

\begin{tabular}{l|l}
\hline \multicolumn{2}{c}{ Internações no último ano } \\
\hline $.0-1$ internação & 0 \\
\hline $.2-3$ internação & 1 \\
\hline . mais de 3 interações & 2 \\
\hline
\end{tabular}

Tempo desta Internação

\begin{tabular}{l|l}
\hline. menos de 10 dias & 0 \\
\hline $.10-30$ dias & 1 \\
\hline. mais de 30 dias & 2 \\
\hline
\end{tabular}

Deambulação

\begin{tabular}{l|l}
\hline . sem auxílio & 0 \\
\hline . com auxílio & 1 \\
\hline . não deambula & 2 \\
\hline
\end{tabular}

\begin{tabular}{l|l}
\multicolumn{2}{c}{ Plegias } \\
\hline . ausentes & 0 \\
\hline . pres. c/ adaptação & 1 \\
\hline . pres. s/ adaptação & 2 \\
\hline
\end{tabular}

Eliminações

\begin{tabular}{l|l}
\hline . sem auxilio & 0 \\
\hline . com auxílio ou sonda & 1 \\
\hline . sem controle esfíncteres & 2 \\
\hline . sondagem intermitente & 3 \\
\hline
\end{tabular}

\begin{tabular}{l|l}
\multicolumn{2}{c}{ Estado Nutricional } \\
\hline . eutrófico & 0 \\
\hline . emagrecido & 1 \\
\hline . caquético & 2 \\
\hline
\end{tabular}

\begin{tabular}{l|l}
\multicolumn{2}{c}{ Higiene } \\
\hline . sem auxílio & 0 \\
\hline . com auxílio & 1 \\
\hline . dependente & 2 \\
\hline
\end{tabular}

SUBTOTAL $1(x 1)$

Obs.

\section{GRUPO 2}

\begin{tabular}{l|l}
\hline \multicolumn{2}{c}{ Alimentação } \\
\hline . sem auxílio & 0 \\
\hline . assistida & 1 \\
\hline . por sonda & 2 \\
\hline . por cateter & 3 \\
\hline
\end{tabular}

\section{Curativos}

\begin{tabular}{l|l}
\hline . ausentes ou simples & \\
\hline . médios & 1 \\
\hline . grandes & 2 \\
\hline . complexos & 3 \\
\hline
\end{tabular}

\begin{tabular}{l|l}
\multicolumn{2}{c}{ Nível de Consciência } \\
\hline . consciente e calmo & 0 \\
\hline . consciente e agitado & 1 \\
\hline . confuso & 2 \\
\hline . comatoso & 3 \\
\hline
\end{tabular}

SUBTOTAL 2 (x2)

\begin{tabular}{c|l}
\hline Totais & \multicolumn{2}{|c}{ Programa } \\
\hline$<8$ & sem indicação Int. \\
\hline 8 a 15 & I.D. c/ visitas de \\
\hline 16 a 20 & I.D. c/ até $6 \mathrm{~h} \mathrm{de}$ \\
\hline 21 a 30 & I.D. c/ até $12 \mathrm{~h} \mathrm{de}$ \\
\hline$>30$ & I.D. c/ ate $24 \mathrm{~h} \mathrm{de}$ \\
\hline
\end{tabular}

TOTAL $1+2+3$
GRUPO 3

\begin{tabular}{l|l}
\hline \multicolumn{2}{c}{ Secreção Pulmonar } \\
\hline . ausente & 0 \\
\hline . peq/mod.quantidade & 1 \\
\hline . abundante & 2 \\
\hline
\end{tabular}

Drenos/Catet./Estomias

\begin{tabular}{l|l}
\hline .ausentes & \\
\hline . presente c/ família apta & 1 \\
\hline . presente c/família & 2 \\
\hline
\end{tabular}

Medicações

\begin{tabular}{l|l}
\hline .VO ou SNE & 0 \\
\hline . IM ou SC 1 ou $2 x d i a$ & 1 \\
\hline . IM ou SC mais $2 x d i a$ & 2 \\
\hline .EV 1 ou $2 \mathrm{x}$ dia & 3 \\
\hline .EV mais $2 \mathrm{x}$ dia & 4 \\
\hline
\end{tabular}

Quadro Clínico

\begin{tabular}{l|l}
\hline . estável & 0 \\
\hline . instabilidade parcial & 1 \\
\hline . instável & 2 \\
\hline
\end{tabular}

Padrão Respiratório

\begin{tabular}{l|l}
\hline . eupnéico & 0 \\
\hline . períodos de dispnéia & 1 \\
\hline . dispnéia constante & 2 \\
\hline . períodos de apnéia & 3 \\
\hline
\end{tabular}

\section{Dependência de O2}

\begin{tabular}{l|l}
\hline . ausente & 0 \\
\hline . parcial (resp. esp.) & 1 \\
\hline . contínua (resp. esp.) & 2 \\
\hline . vent. mecânica interm. & 3 \\
\hline . vent. mecânica contínua & 4 \\
\hline
\end{tabular}

SUBTOTAL $3(x 3)$

Conclusão:

${ }^{6}$ Divulgação autorizada pelo presidente do NEAD, Dr. Ari Bolonhesi 
Anexo IV- Tabela de manutenção em internação domiciliar - Fonte: NEAD, 2004.

\section{TABELA DEMANUTENÇÃOEM INTERNAÇÃO DOMICILIAR - NEAD}

\section{Paciente:}

Convênio:

\section{Datas das Avaliações}

\section{Quadro Clínico}

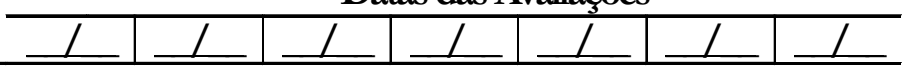

\begin{tabular}{l|l|l|l|l|l|l|l}
\hline . estável & 0 & & & & & & \\
\hline . não estável & 2 & & & & & & \\
\hline Aspirações Traqueais & \multicolumn{1}{|l|}{} & & & \\
\hline . ausentes & 0 & & & & & & \\
\hline . até três aspirações & 1 & & & & & & \\
\hline . três a seis aspirações & 2 & & & & & & \\
\hline .mais de seis aspirações & 4 & & & & & & \\
\hline
\end{tabular}

\section{Sondas/Drenos/Cateteres/Estomias}

. ausentes

. presença com familia apta

. presença sem famillia apta

\section{Procedimentos Técnicos Invasivos}

\begin{tabular}{l|l|l|l|l|l|l|l}
\hline. ausentes & 0 & & & & & & \\
\hline $.1 \mathrm{x}$ dia & 1 & & & & & & \\
\hline $.2 \mathrm{xdia}$ & 2 & & & & & & \\
\hline $.3 \mathrm{x}$ dia & 3 & & & & & & \\
\hline $.4 \mathrm{x}$ dia & 4 & & & & & & \\
\hline. mais de 4x ao dia & 5 & & & & & & \\
\hline
\end{tabular}

\section{Padrão Respiratório}

. eupnéico

. períodos de dispnéia

. dispnéia constante

. períodos de apnéia

\begin{tabular}{|l|l|l|l|l|}
\hline 0 & & & & \\
\hline 1 & & & & \\
\hline 2 & & & &
\end{tabular}

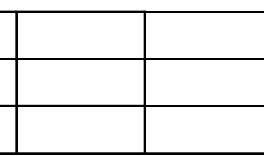

\section{Dependência de $\mathbf{O} 2$}

\begin{tabular}{l|c|c|c|c|c|c|c}
\hline . ausentes & 0 & & & & & & \\
\hline . parcial & 1 & & & & & & \\
\hline . contínua & 2 & & & & & & \\
\hline . ventilação não invasiva & 3 & & & & & & \\
\hline . ventilação invasiva intermitente & 4 & & & & & & \\
\hline . ventilação invasiva contínua & 5 & & & & & & \\
\hline
\end{tabular}

\section{Curativos}

\begin{tabular}{l|l|l|l|l|l|l|l}
\hline . ausentes ou simples & 0 & & & & & & \\
\hline . pequenos & 1 & & & & & & \\
\hline .médios & 2 & & & & & & \\
\hline .grandes/múltiplos & 3 & & & & & & \\
\hline
\end{tabular}

${ }^{7}$ Divulgação autorizada pelo presidente do NEAD, Dr. Ari Bolonhesi 